information on the proposed mechanism will be required before it can be exploited in the design of new high-performance materials. In particular it is unclear whether the observed dark states are indeed populated 'directly' through an indirect transition. The nature of the phonons involved, the possible role of polarons, and the potential for multiple conduction and valence bands still need to be scrutinized. Besides, it is yet to be explained why fast non-radiative recombination does not seem to occur from the dark states, in contrast with the case of the orthorhombic, low-temperature phase of $\mathrm{MAPbI}_{3}$. As shown by Hutter and colleagues, direct access to the mobility of carriers is essential for understanding the details of their dynamics, which, along with a better timeresolution, might be offered by broadband time-resolved terahertz spectroscopy. Further studies using this technique could allow us to unravel the fast processes that occur during the thermalization of photocarriers. They could also provide a handle for identifying the origin of lowfrequency vibrations that are seemingly at the heart of the unique properties of lead halide perovskites.

Jacques-E. Moser is at the Institute of Chemical Sciences \& Engineering of the École Polytechnique
Fédérale de Lausanne, CH-1015 Lausanne, Switzerland.

e-mail:je.moser@epfl.ch

\section{References}

1. Lee, M. M., Teuscher, J., Miyasaka, T., Murakami, T. N. \& Snaith, H. J. Science 338, 643-647 (2012).

2. Kim, H.-S. et al. Sci. Rep. 2, 591 (2012).

3. Saliba, M. et al. Energ Environ. Sci 9, 1989-1997 (2016).

4. Johnston, M. B. \& Herz, L. M. Acc. Chem. Res. 49, 146-154 (2016).

5. Brivio, F., Butler, K. T., Walsh, A. \& van Schilfgaarde, M. Phys. Rev. B 89, 155204 (2014).

6. Motta, C. et al. Nat. Commun. 6, 7026 (2015).

7. Zheng, F., Tan, L. Z., Liu, S. \& Rappe, A. M. Nano Lett. 15, 7794-7800 (2015).

8. Etienne, T., Mosconi, E. \& De Angelis, F. J. Phys. Chem. Lett. 7, 1638-1645 (2016)

9. Hutter, E. M. et al. Nat. Mater. 16, 115-120 (2017).

\title{
A RECIPE FOR GROWTH
}

"Everything is the way it is because it got that way." If D'Arcy Thompson voiced this aphorism, commonly attributed to him, he was probably thinking of how the form of objects can be understood from the growth law that produced them: the idea expounded in his seminal book On Growth and Form, which has its centenary this year. But it is also a statement of historical contingency and path dependence: to understand the way things are, you need to consider the detailed dynamics of formation.

Equilibrium thermodynamics challenges that, asserting instead that the form of a structure corresponds to that of lowest free energy in a pre-existing and predictable energy landscape; dynamics is just the journey to a predetermined destination. But in the real world, complex systems with heterogeneous components often fail to reach that platonic ideal even when they grow under close-to-equilibrium conditions ${ }^{1}$. That might seem to condemn us, in such cases, to making structures riddled with uncontrolled disorder and defects. But might it in fact be possible to control and direct non-equilibrium growth dynamics so as to access a greater diversity of structures than equilibrium offers?

Whitelam et al. have argued that this should be feasible in both one-dimensional polymerizationtype processes ${ }^{2}$, and two- and threedimensional self-assembly ${ }^{3}$. They considered two kinds of building blocks self-assembling stochastically via interactions weak enough to permit disassembly too, under conditions driving the system out of equilibrium. This could apply, for example, to structures self-assembled by colloidal forces, hydrogen-bonding, DNA hybridization, or coordination chemistry in metal-organic frameworks. The out-of-equilibrium driving force determining the growth rate can be tuned to switch between extreme segregation and mixing of the building blocks (Whitelam et al. considered binary mixtures), with some control over the domain size.

Nguyen and Vaikuntanathan have now generalized this approach to propose some design principles for controlled non-equilibrium self-assembly ${ }^{4}$. Their approach is to specify a Hamiltonian for their target structure that represents the equilibrium state of that structure for a set of monomer units with interaction strengths (more precisely, lattice-model coupling constants) rescaled from those that exist in the actual system being studied. The question is then how to tweak the driving forces for self-assembly - in this case, the chemical potentials of the various components, which are proportional to concentration, although other dials should be both necessary and possible - so as to realize this effective Hamiltonian.

Nguyen and Vaikuntanathan show that such trajectories can be calculated in principle for the $1 \mathrm{D}$ and $2 \mathrm{D}$ systems considered by Whitelam et al.,

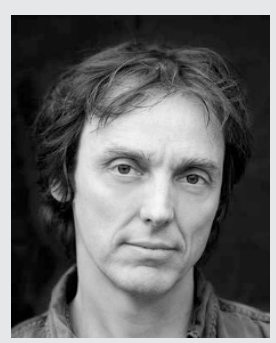

PHILIP BALL

supplying a chemical-potential protocol for achieving a given target structure. They think it should be possible, for example, to switch crystal growth from face-centred cubic to body-centred cubic, or perhaps even to quasicrystalline. The proof will, as ever, be in the experimental pudding.

If such a strategy for controlling non-equilibrium growth via concentration exists, we might wonder whether nature exploits it already. Specific polymer structures there are generally achieved not by stochastic growth but by templating. But might this be the key to, say, nature's apparent ability to direct the polymorphism of calcium carbonate biominerals ${ }^{5}$ ?

References

1. Kim, A. J., Scarlett, R., Biancaniello, P. L., Sinno, T. \& Crocker, J. C. Nat. Mater. 8, 52-55 (2009).

2. Whitelam, S., Schulman, R. \& Hedges, L. Phys. Rev. Lett. 109, 265506 (2012).

3. Whitelam, S., Hedges, L. O. \& Schmidt, J. D. Phys. Rev. Lett. 112, 155504 (2014).

4. Nguyen, M. \& Vaikuntanathan, S. Proc. Natl Acad. Sci. USA http://doi.org/bt6d (2016).

5. Cartwright, J. H., Checa, A. G., Gale, J. D., Gebauer, D. \& Sainz-Díaz, C. I. Angew. Chem. Int. Ed. 51, 11960-11970 (2012). 